\title{
Various micromorphologies and electrochemical properties of polyaniline/ carbon cloth composite nanomaterial were induced for supercapacitors
}

\author{
Xinping He ${ }^{\mathrm{a}}$, Bangning Sun ${ }^{\mathrm{a}}$, Bo Gao ${ }^{\mathrm{b}}$, Anqi Pang ${ }^{\mathrm{a}}$, Hui Suo ${ }^{\mathrm{a}}$, Chun Zhao ${ }^{\mathrm{a}, *}$ \\ a State Key Laboratory on Integrated Optoelectronics, College of Electronic Science and Engineering, Jilin University, Changchun 130012, PR China \\ ${ }^{\mathrm{b}}$ Department of Resources and Environment, Jilin Agriculture University, Changchun 130018, PR China
}

\section{A R T I C L E I N F O}

\section{Article history:}

Received 31 December 2016

Received in revised form 27 February 2017

Accepted 14 March 2017

Available online 15 March 2017

Keywords:

Polyaniline

Carbon cloth

Supercapacitors

Hybrid electrode material

Needle like nanorod

\begin{abstract}
A B S T R A C T
Various nanostructures of polyaniline (PANI) are fabricated on the surface of carbon cloth (CC) by chemical oxidative polymerization. The surface morphologies and electrochemical properties of the PANI/CC hybrid materials are characterized by scanning electron microscope (SEM), water contact angle (CA), cyclic voltammetry (CV) and galvanostatic charge/discharge (GCD) techniques. The morphologies and microstructures of PANI/CC hybrid materials can be controlled by the type of the oxidizing agent. The different morphology of uniformly coated, layer and layer fibrous, ordered needle like PANI built on CC sheet are obtained by using $\mathrm{FeCl}_{3}, \mathrm{H}_{2} \mathrm{O}_{2}, \mathrm{MnO}_{2}$ as the oxidizing agent. The produced hybrid electrode materials are labeled as PANI/CC/FeCl 3 , PANI $/ \mathrm{CC} / \mathrm{H}_{2} \mathrm{O}_{2}, \mathrm{PANI} /$ $\mathrm{CC} / \mathrm{MnO}_{2}$, respectively. We demonstrated that the morphology of the PANI plays an important role in their electrochemical properties. The porous and ordered needle like PANI/CC/MnO 2 exhibits higher specific capacitance (560.5 $\mathrm{F} \mathrm{g}^{-1}$ at $1 \mathrm{~A} \mathrm{~g}^{-1}$ ) and higher energy density (49.78 $\mathrm{Wh} \mathrm{k} \mathrm{g}-1$ at a power density of $400 \mathrm{~W} \mathrm{~kg}^{-1}$ ) than $\mathrm{PANI} / \mathrm{CC} / \mathrm{H}_{2} \mathrm{O}_{2}$ and $\mathrm{PANI} / \mathrm{CC} / \mathrm{FeCl}_{3}$. In addition to this, it also exhibits good long term cycling stability. This work is fully proved that micro-structure can determine the macro-properties by the facts.
\end{abstract}

(c) 2017 Elsevier B.V. All rights reserved.

\section{Introduction}

Energy storage is one of the great challenges in current society. Electrochemical capacitors (ECs), which are also known as supercapacitors, attract great interests because of their faster and higher power energy storage systems, exceptional cycle life, wide thermal operating range and low maintenance cost, filling the gap between batteries (which hold high energy storage) and conventional capacitors (which possess high power output) [1,2]. Activated carbons, transition-metal oxides and conducting polymers are widely used as electrode materials for supercapacitor applications [3-5]. On the basis of the charge storage mechanism, ECs can be divided into electrochemical double layer capacitors (EDLCs) whose capacitance derives from the charge separation at an electrode/electrolyte interface and pseudo-capacitors whose capacitance arises from fast and reversible faradic redox reactions at the surface of active materials [6]. The most EDLCs are carbon based active materials with high specific surface area, transition-metal oxides and conducting polymers are extensively applied to pseudo-capacitor active materials.

Conducting polymers with higher conductivity than the metal oxides and with higher energy density than the carbon material are widely

\footnotetext{
* Corresponding author.

E-mail address: zchun@jlu.edu.cn (C. Zhao).
}

studied [7]. Polyaniline (PANI) is considered as one of the most attractive supercapacitor materials among the conducting polymers, due to its outstanding electrical properties, good environmental stability, low cost and easy synthesis $[8,9]$. However, the lake of stability during cycling is a major shortcoming in the application of PANI [10]. The redox reactions cause the insertion/extraction of counter ions in the molecules chains of PANI which result in a volume change, and the swelling and shrinkage of PANI lead to the degradation of the electrode material during cycling. Thus, the mechanical strength of PANI is the key to improve their long term stability. Over the previous research, much attention has been paid to the synthesis of various structured PANI such as nanosphere, hollow microsphere, nanotube or nanofiber, nanowire, and complex three-dimensional (3D) microstructures [11-14]. In addition to this, the mechanical strength of electrode material can be enhanced by adding various materials to form different hybrid materials [1517]. For example, carbon and conducting polymers hybrid material shows high capacitance and good cycle stability. This is attributed to the synergetic combination of the excellent mechanical properties of carbon materials and high pseudo-capacitance of the PANI [18].

In this paper we present a facile and convenient route to directly fabricate PANI nanomaterial with different morphologies onto the surface of carbon cloth (CC) by chemical oxidative polymerization. The uniformly coated, layer and layer fibrous, ordered needle like PANI are obtained by using $\mathrm{FeCl}_{3}, \mathrm{H}_{2} \mathrm{O}_{2}$, and $\mathrm{MnO}_{2}$ as oxidizing agent, respectively. 
CC was specifically selected as the current collector due to its cost-effectiveness, high conductivity, reasonable chemical stability, and 3D structures with high porosity for PANI nanomaterial growth. The polyaniline/ carbon cloth (PANI/CC) hybrid nanomaterial with various microstructures is investigated by scanning electron microscope (SEM), water contact angle (CA), cyclic voltammetry (CV) and galvanostatic charge/discharge (GCD) tests. The experimental results indicate that the PANI/CC composite nanomaterial owns ordered nanoarchitectured structure and excellent capacitive performance, a specific capacitance as high as $1121 \mathrm{~F} \mathrm{~g}^{-1}$ at a current density of $2 \mathrm{~A} \mathrm{~g}^{-1}$ is observed in the electrode, as well as good rate performance and cyclic stability.

\section{Experimental}

\subsection{Materials and reagents}

Carbon cloth (300 $\mathrm{g} \mathrm{m}^{-2}, 1 \mathrm{~mm}$ thickness, carbon content: 93\%) was purchased from Shuangpeng carbon cloth factory (Jilin, P.R. China). And for performance test experiment, carbon cloth was chopped into pieces with dimension of $10 \mathrm{~mm} \times 10 \mathrm{~mm}$. Then the CC was treated in $25 \%$ hydrochloric acid at $40{ }^{\circ} \mathrm{C}$ for $20 \mathrm{~min}$, cleaned consecutively in acetone and deionized water several times. All other chemicals were obtained from a local chemical agent. Aniline monomer was distilled under reduced pressure and the resulting colorless liquid was kept in the dark at $4{ }^{\circ} \mathrm{C}$ prior to use. Manganese dioxide, ferric trichloride, hydrogen peroxide, sulfuric acid (98\%) and hydrochloric acid (35\%) were used without any further purification.

\subsection{Fabrication of various $P A N I / C C$}

PANI was modified on the CC sheets by chemical oxidative polymerization. The various PANI/CC hybrid material were prepared as follow: aniline $(0.1 \mathrm{M})$ was uniformly dispersed into $(1 \mathrm{M})$ hydrochloric acid by ultrasonic processing for $5 \mathrm{~min}$. A variety of manganese dioxide solution $(0.1 \mathrm{M}, 0.2 \mathrm{M}, 0.4 \mathrm{M}, 0.6 \mathrm{M}, 0.8 \mathrm{M}, 1 \mathrm{M})$ as oxidizing agent were also prepared in (1 M) hydrochloric acid and then ultrasonic treatment to make sure manganese dioxide particles dispersed homogeneously. Aniline and manganese dioxide solution were mixed (volume1:1) in a centrifugal tube containing a piece of pre-treated CC sheet. The polymerization was carried out in an ice-water bath at $0{ }^{\circ} \mathrm{C}$. After polymerization for $24 \mathrm{~h}$, the synthetic PANI/CC hybrid materials were washed with distilled water and ethanol three times to remove excess acid, possible oligomers and inorganic ion, and then dried at $50{ }^{\circ} \mathrm{C}$ for $12 \mathrm{~h}$. The PANI/CC hybrid materials were also synthesized using ferric trichloride and hydrogen peroxide as oxidizing agent via the similar procedure. The PANI/CC hybrid materials prepared using manganese dioxide, hydrogen peroxide and ferric trichloride as oxidative agent were marked as $\mathrm{PANI} / \mathrm{CC} / \mathrm{MnO}_{2}, \mathrm{PANI} / \mathrm{CC} / \mathrm{H}_{2} \mathrm{O}_{2}$ and $\mathrm{PANI} / \mathrm{CC} / \mathrm{FeCl}$, respectively. The typical mass loadings of the active material on the carbon cloth were around $1.2 \mathrm{mg} \mathrm{cm}^{-2}$.

\subsection{Characterization}

The morphologies of the pristine CC and PANI/CC hybrid materials were characterized by a field-emission SEM (FE-SEM, JSM-6700F, JEOL, and Japan) at $5 \mathrm{kV}$. Based on static water contact angle measurement, the surface wetting property of the pristine CC and PANI/CC hybrid materials were evaluated by a Contact Angle System OCA 20 (Data Physics Instruments GmbH, Germany).

Some electrochemical characterization experiments were performed in a three-electrode system with a $\mathrm{CHI}$ 660D electrochemical workstation. The pristine CC and PANI/CC hybrid materials were used as working electrodes. A platinum plate was used as counter electrode and a saturated calomel electrode (SCE) was used as reference electrode. $1 \mathrm{M} \mathrm{H}_{2} \mathrm{SO}_{4}$ was used as electrolyte. In order to evaluate the electrochemical capacitance performance of the PANI/CC hybrid materials,
$\mathrm{CV}$ and GCD method were employed. For the pristine CC, PANI/CC/ $\mathrm{MnO}_{2}$ and $\mathrm{PANI} / \mathrm{CC} / \mathrm{FeCl}_{3}, \mathrm{CV}$ was carried out in a potential range of 0.1 to $1.5 \mathrm{~V}$ (vs. SCE) at varying scan rates of $10,20,30,40$ and $50 \mathrm{mV} \mathrm{s}^{-1}$. The $\mathrm{CV}$ potential range of $\mathrm{PANI} / \mathrm{H}_{2} \mathrm{O}_{2}$ is 0.1 to $1.6 \mathrm{~V}$ (vs. $\mathrm{SCE}$ ). GCD properties were measured at step increasing current densities of 1 to $8 \mathrm{~A} \mathrm{~g}^{-1}$ at the operating voltage from 0.15 to $0.95 \mathrm{~V}$.

Battery testing system (Neware) was used to measure the cycle life of the prepared hybrid materials. A platinum plate was used as the counter electrode. The prepared hybrid material was used as working electrode. $1 \mathrm{M} \mathrm{H}_{2} \mathrm{SO}_{4}$ was used as electrolyte. In order to eliminate $\mathrm{O}_{2}$ gas in the electrolyte, the $\mathrm{H}_{2} \mathrm{SO}_{4}$ solution was charged pure $\mathrm{N}_{2}$ for $10 \mathrm{~min}$ before the electrochemical measurements.

\section{Results and discussion}

3.1. Surface morphology and hydrophilicity of the PANI modified carbon cloth

Ordered nanoarchitectured structure of conducting polymer can create new opportunities that lead to better performance in many already established areas, such as batteries, sensors, actuators, catalysts, electro-optic and electrochromic devices $[19,20]$. Therefore, in order to satisfy the needs of both researches and applications, reliable and scalable synthetic methods for conducting polymers to obtain various nanostructures are widely studied. Here, we use an easy and effective method to fabricate three different nanostructures of PANI/CC hybrid electrode materials.

Fig. 1 shows micrographs of carbon cloth sheets before and after modification of PANI. The XRD measurement fully proved the successful synthesis of polyaniline (details in Supporting information S2). We could observe that the surface of the original CC is smooth (Fig. 1a). Fig. 1b, c and d display PANI/CC hybrid electrode material with manganese dioxide, ferric trichloride and hydrogen peroxide as oxidizing agent, respectively. For PANI/CC/FeCl 3 , the PANI nanoparticles are uniformly and intensively coated on the surface of CC skeleton and the diameter of the nanoparticles are about 20 to $30 \mathrm{~nm}$. It looks like the dense coating composed of nanoparticles. For PANI/CC $/ \mathrm{H}_{2} \mathrm{O}_{2}$, layer after layer PANI nanofibers covered on the surface of CC skeleton and the diameter of the nanofibers are about 30 to $50 \mathrm{~nm}$. The nanofibers are mutual crisscross with some pores between them, and some of them aggregated together. For PANI/CC/MnO very different structure, the ordered independent needle like PANI nanowires with about $30 \mathrm{~nm}$ diameter aligned vertically on the CC skeleton. There are many pores between the nanowires. Through comparison with $\mathrm{PANI} / \mathrm{CC} / \mathrm{FeCl}{ }_{3}$ and $\mathrm{PANI} / \mathrm{CC} / \mathrm{H}_{2} \mathrm{O}_{2}$, the $\mathrm{PANI} / \mathrm{CC} / \mathrm{MnO}_{2}$ shows a highly porous and orderly structure, which could provide both an higher efficient contact surface area (details in Supporting information S1) for the intercalation/deintercalation of protons into/out of active materials and a shorter path length for electronic transport and the fast access of electrolyte ions.

On the basis of the above experimental results, the formation of PANI/CC hybrid electrode materials is depicted schematically in Fig. 2. The cleaned CC $(10 \mathrm{~mm} \times 10 \mathrm{~mm})$ is immersed in the centrifuge tube containing aniline hydrochloric acid for polymerization, aniline monomers are adsorbed on the surface of CC sheet. The active functional groups $(-\mathrm{OH}$ and $-\mathrm{COOH})$ on the surface of $\mathrm{CC}$ sheets promoted the adsorption of aniline monomers onto the CC sheet. When the oxidations are added into the above centrifuge tube, the aniline monomers absorbed on the surface of $\mathrm{CC}$ sheet are easily activated and begin to polymerize from active sites via heterogeneous nucleation. These active sites minimize the interfacial energy between the bulky solution and solid substrates. During the growth stage of PANI on the CC sheet, with the different conformation of PANI chains, the degree of conjugation of PANI is totally different under different oxidative agent. Beyond that, the expanded degree of the PANI chains changes frequently, 


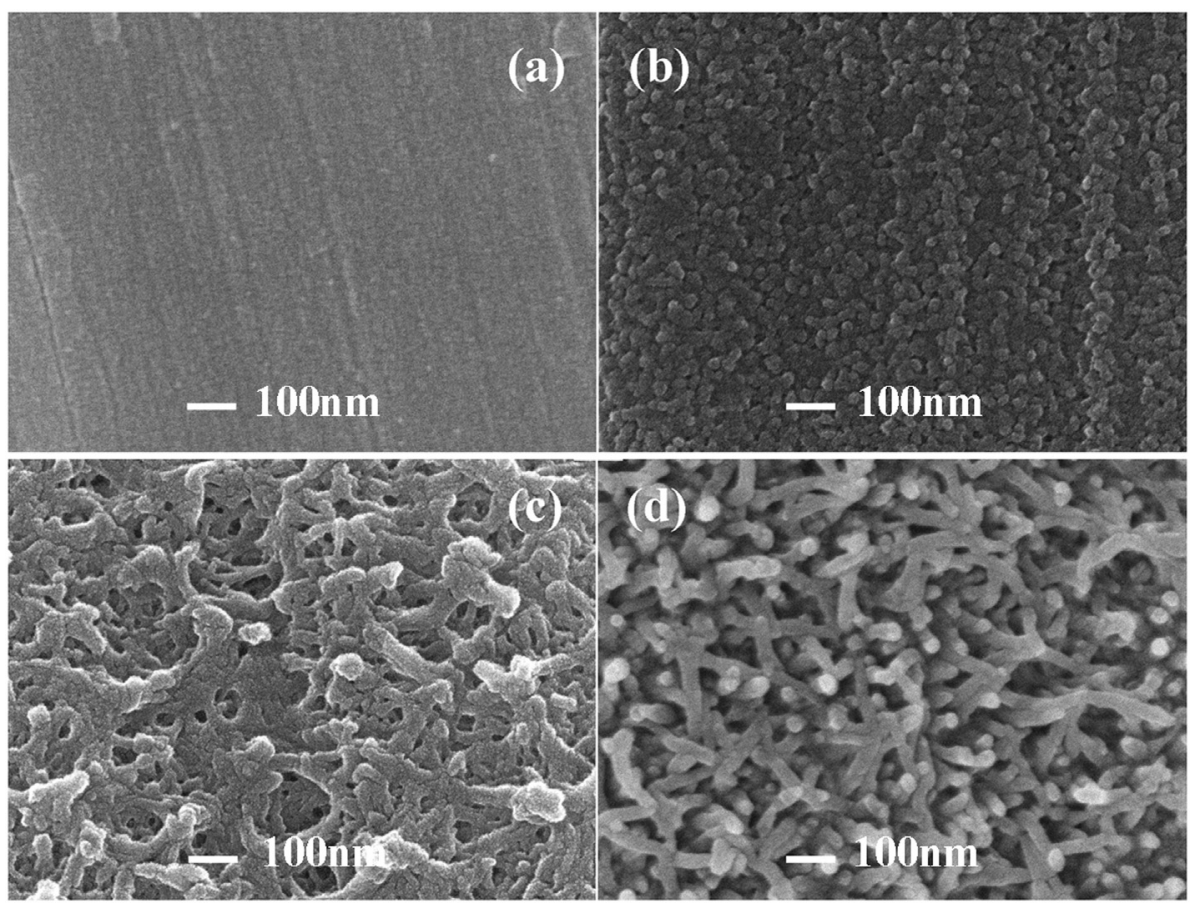

Fig. 1. $\mathrm{SEM}$ images of $\mathrm{CC}, \mathrm{PANI} / \mathrm{CC} / \mathrm{FeCl}{ }_{3}, \mathrm{PANI} / \mathrm{CC} / \mathrm{H}_{2} \mathrm{O}_{2}$ and $\mathrm{PANI} / \mathrm{CC} / \mathrm{MnO}_{2}$ hybrid electrode materials: (a)CC; (b) $\mathrm{PANI} / \mathrm{CC} / \mathrm{FeCl}$; (c) $\mathrm{PANI} / \mathrm{CC} / \mathrm{H}_{2} \mathrm{O}_{2}$; (d) $\mathrm{PANI} / \mathrm{CC} / \mathrm{MnO}{ }_{2}$.

which leads the various stacking ways of PANI and further makes PANI possess different deposited structures.

The surface wetting property of the prepared hybrid electrode material is evaluated by the measurement of water contact angle. As we know, the surface water contact angle is related to the surface morphology and chemical composition. Fig. 3 shows the photo images of water contact angle of the carbon cloth that prepared without and with PANI decorated. We could observe that the pristine CC exhibited a static water contact angle of $137-146^{\circ}$, indicating the pristine CC is surface hydrophobicity. However, upon the modification with PANI, the water drop in the measurement was completely and rapidly adsorbed into the PANI/CC hybrid materials. Such behavior attested that the surface
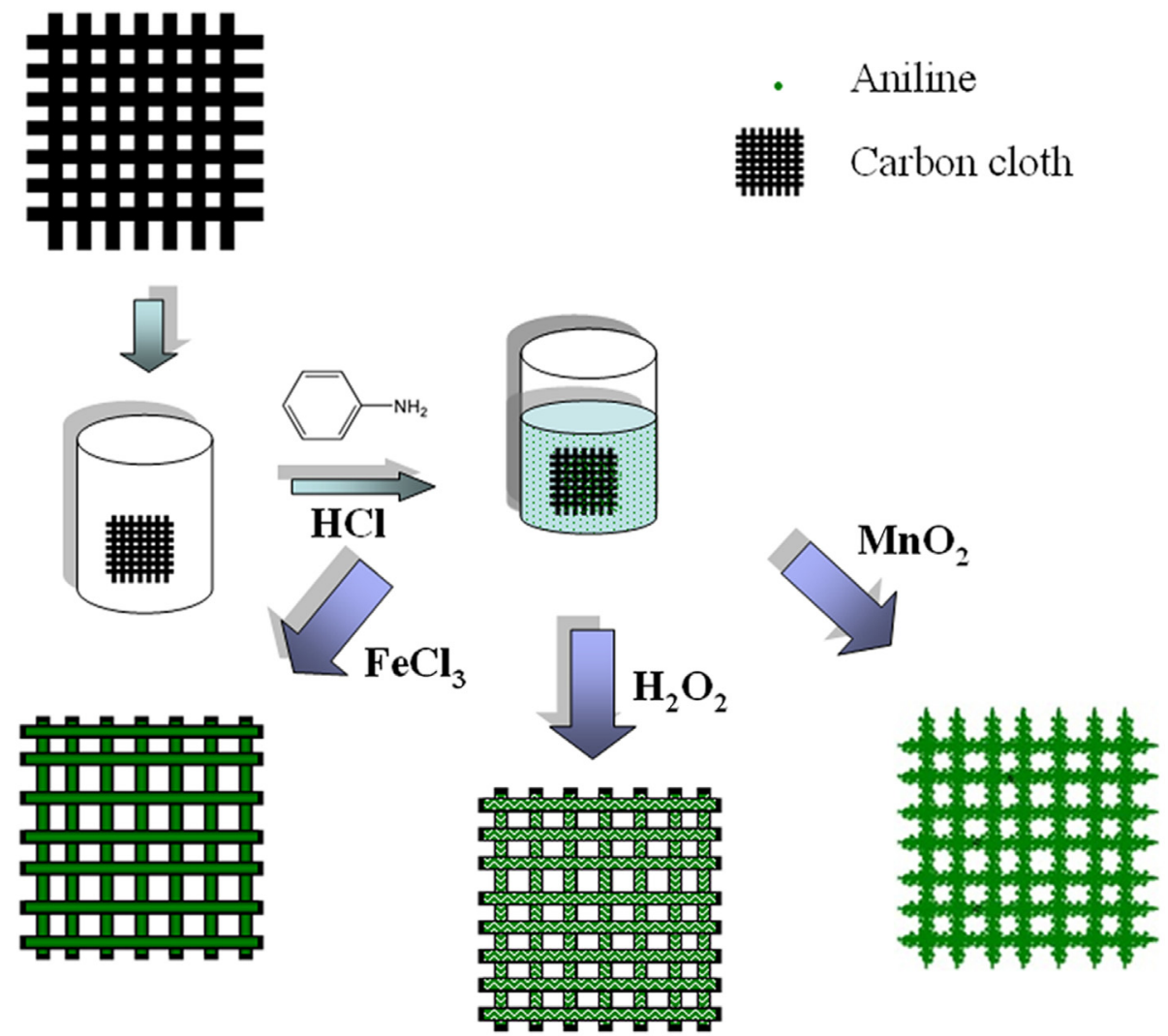

Fig. 2. Schematic illustration of the process for preparation of PANI/CC hybrid electrode materials using different oxidant. 

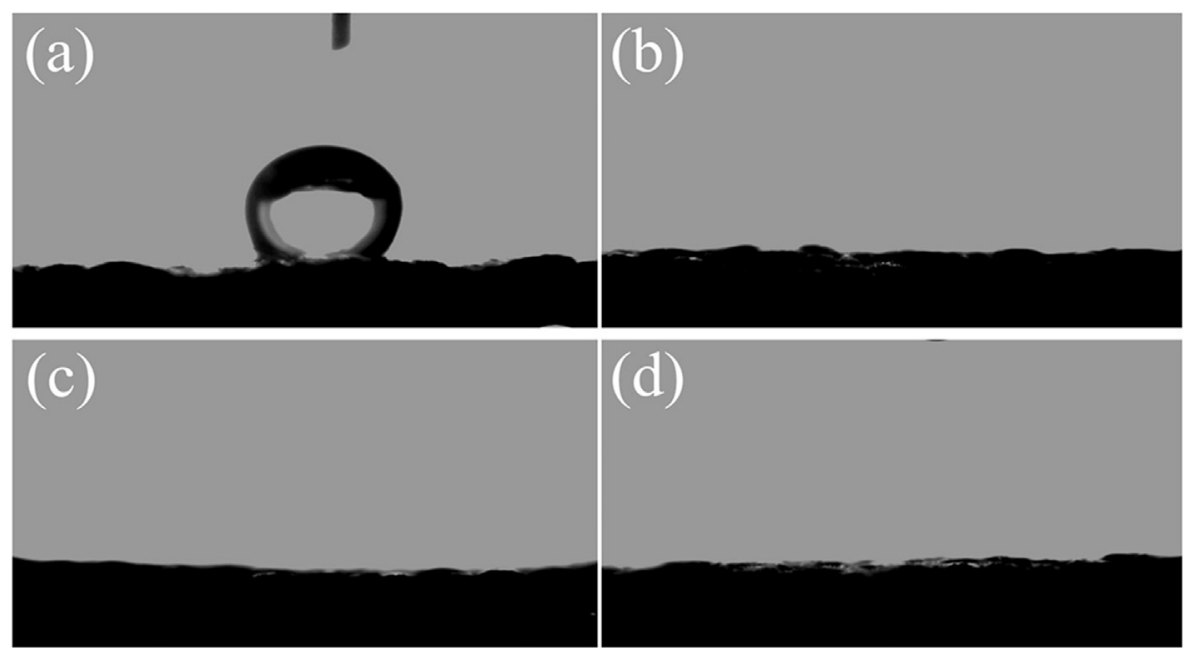

Fig. 3. Photo images of contact angle measurement with $\mathrm{CC}$ and $\mathrm{PANI} / \mathrm{CC}$ hybrid electrode materials surface. (a) $\mathrm{CC}$; (b) $\mathrm{PANI} / \mathrm{CC} / \mathrm{FeCl}{ }_{3}$; (c) $\mathrm{PANI} / \mathrm{CC} / \mathrm{H}_{2} \mathrm{O}_{2}$; (d) $\mathrm{PANI} / \mathrm{CC} / \mathrm{MnO}{ }_{2}$.

properties changed from hydrophobic to hydrophilic resulting from the deposition of PANI. The hydrophilic surface is advantageous to the electrolyte fully contact to the surface of active material.

\subsection{Electrochemical properties}

In order to evaluate the electrochemical characteristics of the samples, CV and the GCD experiments are performed in a conventional three-electrode system. The CV measurements (the potential window for CC, $\mathrm{PANI} / \mathrm{CC} / \mathrm{FeCl}_{3}$ and $\mathrm{PANI} / \mathrm{CC} / \mathrm{MnO}_{2}$ is from 0.1 to 1.5 versus SCE, for PANI/CC $/ \mathrm{H}_{2} \mathrm{O}_{2}$ is from 0.1 to 1.6 ) of the pristine and modified $\mathrm{CC}$ sheets at scan rate of $50 \mathrm{mV} \mathrm{s}^{-1}$ are illustrated in Fig. 4a. As can be observed, for the pristine CC sheet, no pair of redox peaks appear which exhibits typical double-layer capacitance characteristics; in contrast, three pairs of redox peaks of PANI modified CC sheets are evident, which are related to various oxidation states of PANI. $\mathrm{A} / \mathrm{A}^{\prime}$ and $\mathrm{C} / \mathrm{C}^{\prime}$ are attributed to the redox of PANI molecules and $\mathrm{B} / \mathrm{B}^{\prime}$ is the redox of benzoquinone and aminoquinone species [21-23]. The CV curve areas of the PANI modified CC sheet are almost orders of magnitude higher than that of the pristine $\mathrm{CC}$ sheet, which reveals the main capacitive performance of the PANI/CC hybrid electrode material is derived from PANI rather than from CC. It also can be noted, the CV curve area of the PANI/ $\mathrm{CC} / \mathrm{MnO}_{2}$ is a little larger than that of the PANI/CC/ $\mathrm{H}_{2} \mathrm{O}_{2}$ and much larger than that of the $\mathrm{PANI} / \mathrm{CC} / \mathrm{FeCl}_{3}$, which indicates that the specific
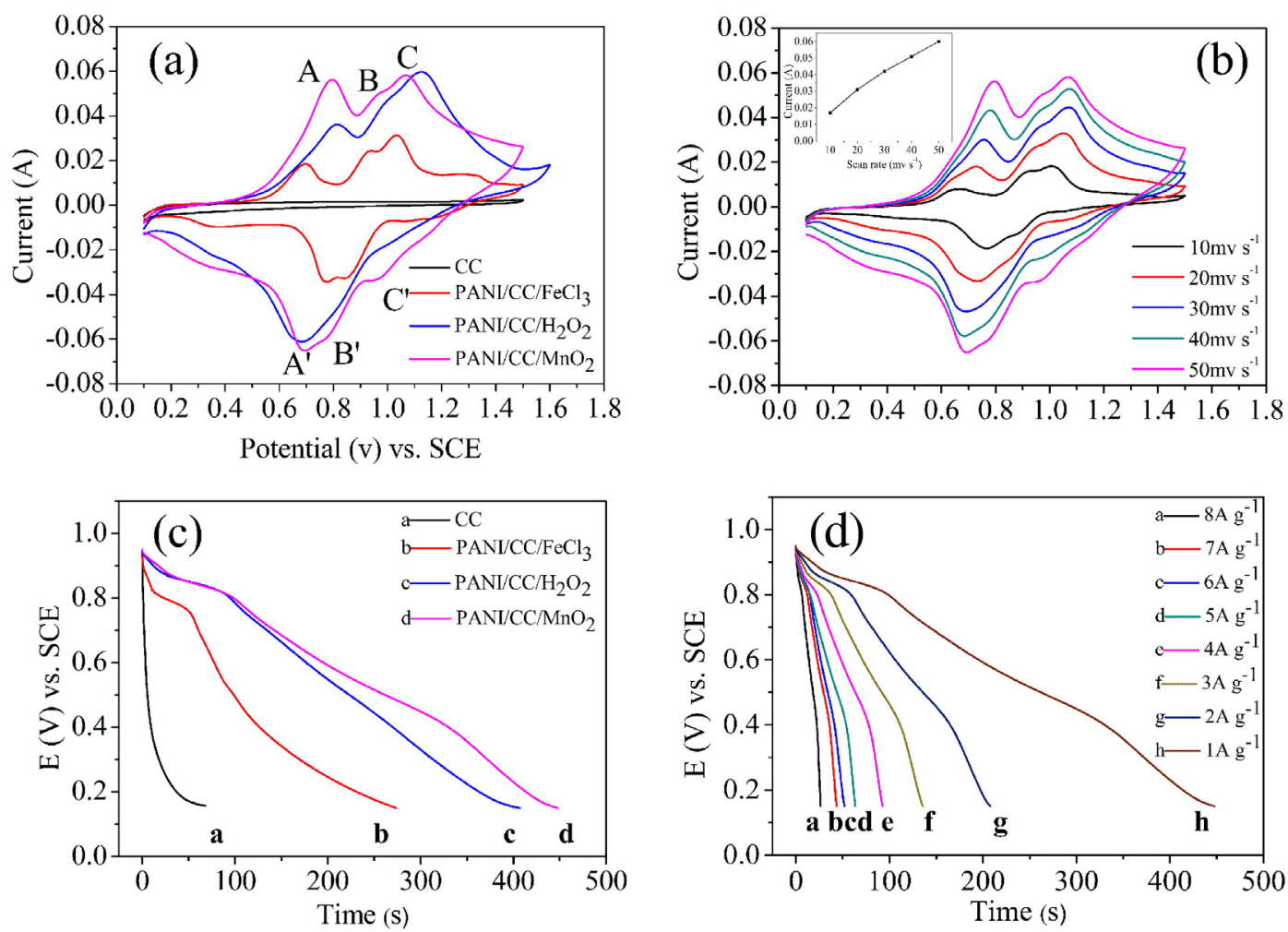

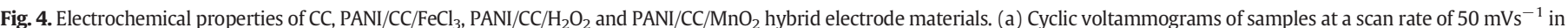

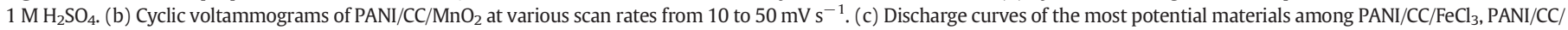

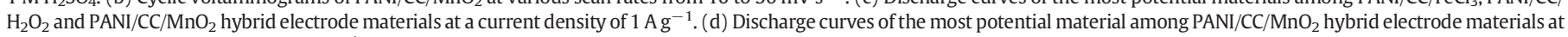
various current density from 1 to $8 \mathrm{~A} \mathrm{~g}^{-1}$. 
capacitance of PANI/CC hybrid electrode material as follows: PANI/CC/ $\mathrm{MnO}_{2}>\mathrm{PANI} / \mathrm{CC} / \mathrm{H}_{2} \mathrm{O}_{2}>\mathrm{PANI} / \mathrm{CC} / \mathrm{FeCl}_{3}$. Two main factors may lead to the result. Firstly, the ordered needle-like PANI structure of PANI/CC/ $\mathrm{MnO}_{2}$ provides large specific surface area, it not only can benefit the intercalation/deintercalation of protons into/out of active materials, but also can shorten the ions diffusion lengths. Secondly, the highly porous structure coming from needle-like PANI nanowires is convenient for the fast access of ions from solution to the each PANI nanowires and ensures the efficient utilization of the active materials. Hence the CV curve of the $\mathrm{PANI} / \mathrm{CC} / \mathrm{MnO}_{2}$ hybrid electrode material covers the largest area. Fig. $4 \mathrm{~b}$ shows the CVs of PANI/CC/MnO 2 at scan rate of $10,20,30,40$ and $50 \mathrm{mV} \mathrm{s}^{-1}$, respectively. The plots of the current density versus the different scan rates for the highest oxidation peaks are given in the inset of Fig. 4b. It displays that the current density increases with an increase in scan rate. This implies the redox reaction is not kinetically limited at least within the scan rates of 10 to $50 \mathrm{mV} \mathrm{s}^{-1}$.

Fig. $4 \mathrm{c}$ reveals the galvanostic discharge curves of the pristine $\mathrm{CC}$, $\mathrm{PANI} / \mathrm{CC} / \mathrm{FeCl}_{3}, \mathrm{PANI} / \mathrm{CC} / \mathrm{H}_{2} \mathrm{O}_{2}$ and $\mathrm{PANI} / \mathrm{CC} / \mathrm{MnO}_{2}$ at current density of $1 \mathrm{Ag}^{-1}$.

Their specific capacitance (Cs) can be calculated by Eq. (1) [24,25]:

$C S=\frac{c}{m}=\frac{I \times \mathrm{t}}{\Delta V \times m}$

where, Cs is specific capacitance ( $\left.\mathrm{F} \mathrm{g}^{-1}\right)$, I is the discharge current (A), $\Delta \mathrm{V}$ is potential drop during discharge in $\mathrm{V}$, and $\mathrm{m}$ is the active material in $\mathrm{g}$. The discharge specific capacitance of $\mathrm{PANI} / \mathrm{CC} / \mathrm{MnO}_{2}, \mathrm{PANI} / \mathrm{CC} /$ $\mathrm{H}_{2} \mathrm{O}_{2}$ and $\mathrm{PANI} / \mathrm{CC} / \mathrm{FeCl}_{3}$ is $560.5 \mathrm{~F} \mathrm{~g}^{-1}, 509 \mathrm{~F} \mathrm{~g}^{-1}$ and $342.25 \mathrm{~F} \mathrm{~g}^{-1}$ at a current density of $1 \mathrm{~A} \mathrm{~g}^{-1}$. From this, we could be easier to get the conclusion that the discharge performance of $\mathrm{PANI} / \mathrm{CC} / \mathrm{MnO}_{2}$ is better than $\mathrm{PANI} / \mathrm{CC} / \mathrm{H}_{2} \mathrm{O}_{2}$ and $\mathrm{PANI} / \mathrm{CC} / \mathrm{FeCl}_{3}$ at the same current density. Because of the ordered needle like nanowire structure effectively increase the utilization of the active material and shorten the transmission route of the electron and ions than the uniform coating and layer after layer structure. The discharge curves of the PANI/CC/ $\mathrm{MnO}_{2}$ hybrid electrode material at various current densities of $1,2,3,4,5,6,7$ and $8 \mathrm{~A} \mathrm{~g} \mathrm{~g}^{-1}$ are exhibited in Fig. $4 \mathrm{~d}$. The specific capacitance decreases with the increase of the discharge current density. From 1 to $8 \mathrm{Ag}^{-1}$, the discharge capacitance of the PANI/CC/ $\mathrm{MnO}_{2}$ hybrid electrode material reduces from $560.5 \mathrm{~F} \mathrm{~g}^{-1}$ to $264.5 \mathrm{Fg}^{-1}$, a decrease by $52.8 \%$ of the initial capacitance. This result is attributed to the fact that the redox reaction rates and the charge diffusion cannot follow the current density increase. A more stable capacitance of PANI nanomaterial is worth to be breakthrough in future research.

Fig. 5a, b and c shows the galvanostatic discharge curves of PANI/CC/ $\mathrm{FeCl}_{3}, \mathrm{PANI} / \mathrm{CC} / \mathrm{H}_{2} \mathrm{O}_{2}$ and $\mathrm{PANI} / \mathrm{CC} / \mathrm{MnO}_{2}$ with different concentration $\left(0.1,0.2,0.4,0.6,0.8\right.$ and $\left.1 \mathrm{~mol} \mathrm{~L}^{-1}\right)$ of the corresponding oxidants at current density of $1 \mathrm{~A} \mathrm{~g}^{-1}$. The calculated capacitance of the electrodes at the current density of $1 \mathrm{Ag}^{-1}$ is plotted in Fig. $5 \mathrm{~d}$. From those results, we get the most suitable oxidant concentration for the different oxidants $\left(\mathrm{FeCl}_{3}, \mathrm{H}_{2} \mathrm{O}_{2}\right.$ and $\left.\mathrm{MnO}_{2}\right)$ to reach the maximum discharge specific capacitance of the various morphology PANI/CC hybrid electrode materials. The most suitable oxidant concentration for the PANI/CC/FeCl 3 , $\mathrm{PANI} / \mathrm{CC} / \mathrm{H}_{2} \mathrm{O}_{2}$ and $\mathrm{PANI} / \mathrm{CC} / \mathrm{MnO}_{2}$ is $0.8 \mathrm{M}, 0.8 \mathrm{M}$ and $0.4 \mathrm{M}$ respectively. Their discharge specific capacitance at a current density of $1 \mathrm{~A} \mathrm{~g}^{-1}$ is $560.5 \mathrm{~F} \mathrm{~g}^{-1}, 509 \mathrm{Fg}^{-1}$ and $342.25 \mathrm{~F} \mathrm{~g}^{-1}$. In the following study, we just focus on these three materials.

The calculated capacitance of the three electrodes at various discharge current densities are plotted in Fig. 6a. Notably, the specific capacitance of PANI/CC hybrid material decreases with the increase in discharge current densities. However, all the specific capacitance of $\mathrm{PANI} / \mathrm{CC} / \mathrm{MnO}_{2}$ exhibits a much higher discharge capacitance than $\mathrm{PANI} / \mathrm{CC} / \mathrm{H}_{2} \mathrm{O}_{2}$ and $\mathrm{PANI} / \mathrm{CC} / \mathrm{FeCl}_{3}$. And the specific capacitance of $\mathrm{PANI} / \mathrm{CC} / \mathrm{MnO} 2$ is $560.5 \mathrm{~F} \mathrm{~g}^{-1}, 520 \mathrm{~F} \mathrm{~g}^{-1}, 508.75 \mathrm{~F} \mathrm{~g}^{-1}, 463.75 \mathrm{~F} \mathrm{~g}^{-1}$, $396.75 \mathrm{~F} \mathrm{~g}^{-1}, 390 \mathrm{~F} \mathrm{~g}^{-1}, 381.94 \mathrm{~F} \mathrm{~g}^{-1}$ and $264.5 \mathrm{~F} \mathrm{~g}^{-1}$ at the discharge current densities of $1,2,3,4,5,6,7$, and $8 \mathrm{~A} \mathrm{~g}^{-1}$, respectively. By
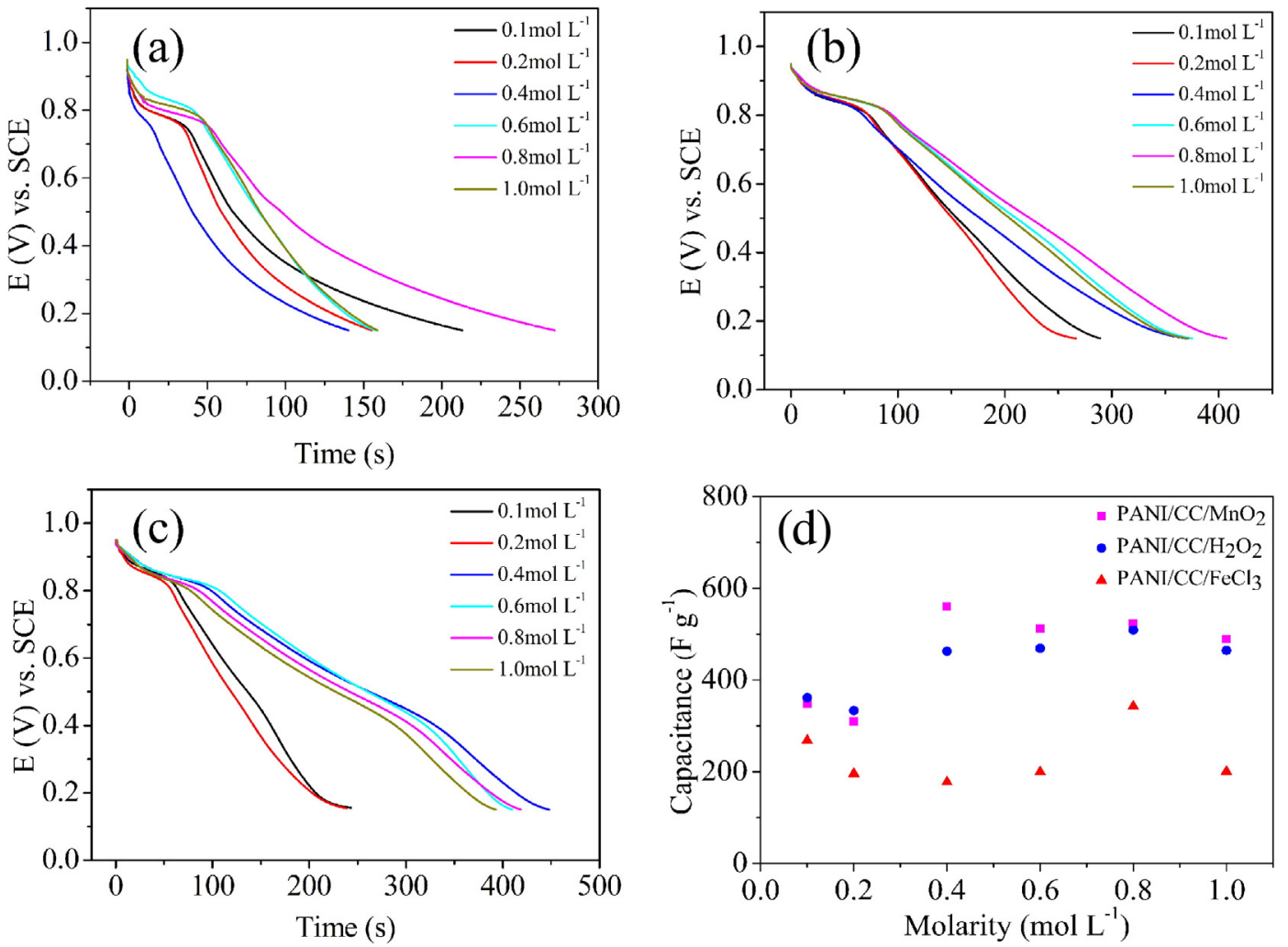

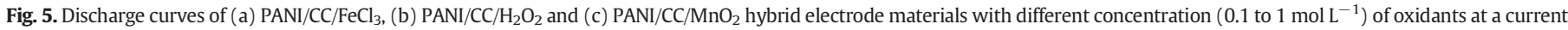

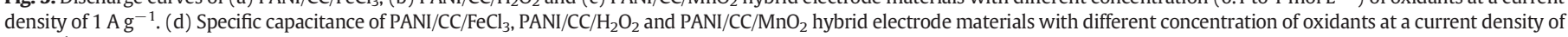
$1 \mathrm{~A} \mathrm{~g}^{-1}$. 

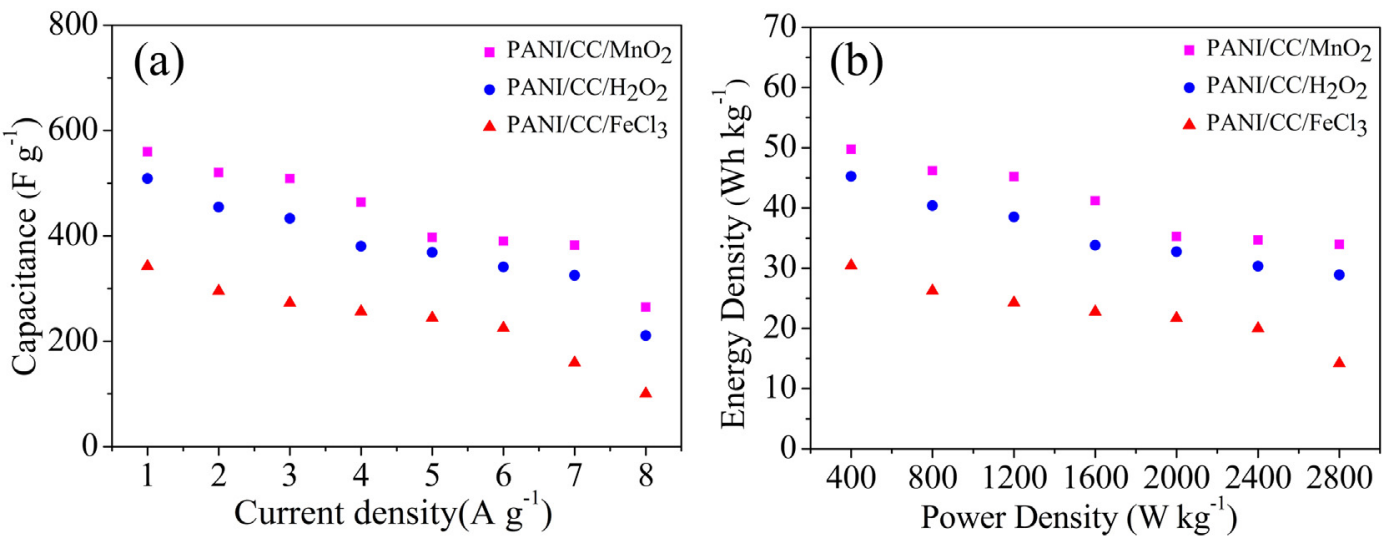

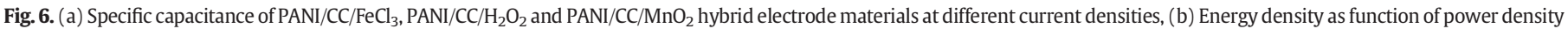
in $1.0 \mathrm{M} \mathrm{H}_{2} \mathrm{SO}_{4}$ electrolyte.

contrast, the specific capacitance of $\mathrm{PANI} / \mathrm{CC} / \mathrm{H}_{2} \mathrm{O}_{2}$ and $\mathrm{PANI} / \mathrm{CC} / \mathrm{FeCl}{ }_{3}$ is $509 \mathrm{~F} \mathrm{~g}^{-1}, 454.48 \mathrm{~F} \mathrm{~g}^{-1}, 433.13 \mathrm{~F} \mathrm{~g}^{-1}, 380.1 \mathrm{~F} \mathrm{~g}^{-1}, 368.38 \mathrm{~F} \mathrm{~g}^{-1}$, $340.73 \mathrm{~F} \mathrm{~g}^{-1}, 324.84 \mathrm{~F} \mathrm{~g}^{-1}, 210.5 \mathrm{~F} \mathrm{~g}^{-1}$ and $342.25 \mathrm{~F} \mathrm{~g}^{-1}$,

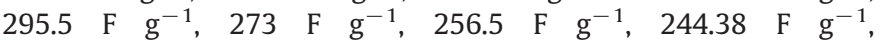
$225 \mathrm{~F} \mathrm{~g}^{-1} .159 .25 \mathrm{~F} \mathrm{~g}^{-1} .100 \mathrm{~F} \mathrm{~g}^{-1}$ respectively. These results fully show the excellent capacitance and rate performance of PANI/CC/ $\mathrm{MnO}_{2}$ hybrid electrode material.

Energy and power densities are two key factors for evaluating the practical applications of electrochemical supercapacitors. The energy density and power density were calculated according to Eqs. (2) and (3) [26], respectively.

$E=\frac{1 \times C s \times \Delta V^{2}}{2 \times 3.6}$

$P=\frac{3600 \times E}{\Delta t}$

where Cs is the specific capacitance calculated from discharge time in $\mathrm{F} \mathrm{g}^{-1}, \Delta \mathrm{V}$ is potential drop during discharge in $\mathrm{V}, \mathrm{t}$ is the discharge time in $\mathrm{s}, \mathrm{E}$ is the energy density of the electrode material in $\mathrm{Wh} \mathrm{kg}^{-1}$ and $\mathrm{P}$ is power density of the electrode material in $\mathrm{W} \mathrm{kg}^{-1}$. The results of the hybrid electrode materials are presented as Ragone plots in Fig. 6b. Obviously, the $\mathrm{PANI} / \mathrm{CC} / \mathrm{MnO}_{2}$ hybrid material displays higher energy density than $\mathrm{PANI} / \mathrm{CC} / \mathrm{H}_{2} \mathrm{O}_{2}$ and $\mathrm{PANI} / \mathrm{CC} / \mathrm{FeCl}$ hybrid material, which is attributed to the needle like nanorod structure

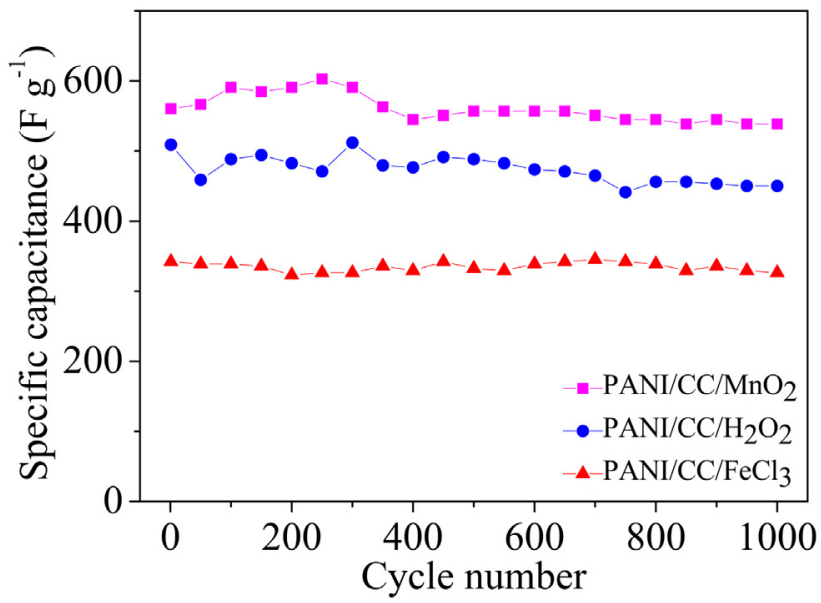

Fig. 7. Variation of specific capacitance as a function of cycle number for $\mathrm{PANI} / \mathrm{CC} / \mathrm{FeCl}_{3}$, $\mathrm{PANI} / \mathrm{CC} / \mathrm{H}_{2} \mathrm{O}_{2}$ and $\mathrm{PANI} / \mathrm{CC} / \mathrm{MnO}_{2}$ hybrid electrode matreials in the $1 \mathrm{M} \mathrm{H}_{2} \mathrm{SO}_{4}$ electrolyte at a discharge current density of $1 \mathrm{~A} \mathrm{~g}^{-1}$. increasing the electrical performance of the material. The PANI/CC/ $\mathrm{MnO}_{2}$ hybrid material has the highest $\mathrm{E}$ at a relatively lower $\mathrm{P}$ ( $\mathrm{E}$ is 49.78 $\mathrm{Wh} \mathrm{kg}^{-1}$ at $\mathrm{P}$ of $400 \mathrm{~W} \mathrm{~kg}{ }^{-1}$ ), while PANI/CC $/ \mathrm{H}_{2} \mathrm{O}_{2}$ and $\mathrm{PANI} / \mathrm{CC} / \mathrm{FeCl}_{3}$ hybrid material exhibit a lower $\mathrm{E}$ (45.24 Wh kg $\mathrm{Wh}^{-1}$ and $30.42 \mathrm{Wh} \mathrm{kg}^{-1}$ ) at the same P. The energy density of PANI/CC/ $\mathrm{MnO}_{2}$ hybrid material can remain $23.51 \mathrm{Wh} \mathrm{kg}^{-1}$ when the power density is increased to $3200 \mathrm{~W} \mathrm{~kg}^{-1}$. This excellent performance is also superior to some reported PANI hybrid materials.

To further evaluate the cycle life of the samples, galvanostatic charge-discharge measurements are carried out in $1 \mathrm{M} \mathrm{H}_{2} \mathrm{SO}_{4}$ electrolyte at a current density of $1 \mathrm{~A} \mathrm{~g}^{-1}$ for 1000 cycles, as shown in Fig. 7. There is a slight increase in the discharge specific capacitance values in the first cycles of the PANI/CC/MnO 2 electrode material, thereafter, the specific capacitance decreases with a further increase of the cycle times. The capacitance retention of $\mathrm{PANI} / \mathrm{CC} / \mathrm{MnO}_{2}$ can retain $96.2 \%$ of its original value after 1000 cycles. Just 3.8\% decline which almost occurred in the first 400 circles and then nearly keep the same capacitance. In addition to this, $\mathrm{PANI} / \mathrm{CC} / \mathrm{H}_{2} \mathrm{O}_{2}$ and $\mathrm{PANI} / \mathrm{CC} / \mathrm{FeCl}_{3}$ also possesses good cycle stability which keeps $88.4 \%$ and $95.4 \%$ of the original value, respectively. At the cycle life test, the dope or dedope of $\mathrm{H}^{+}$ into or from the PANI chains results in the swelling and shrinkage of the nanostructure conducting polymer, which is the significant factor to generate the specific capacitance decrease.

\section{Conclusion}

Various nanostructure PANI are controllably, stably and binder freely modified on the surface of CC by chemical oxidative polymerization. With the modification of a rough nanosize PANI film, the hydrophobic surface of CC becomes hydrophilic. And then, the active material can be readily accessed and wetted by the electrolyte. From this study, the morphologies of PANI/CC hybrid material are successfully controlled by using different oxidative agent. The morphologies of the PANI/CC nanocomposites are uniform coated, layer and layer fibrous and ordered needle like, respectively. The hybrid electrode materials with different structure exhibit different electrochemical property. The needle like $\mathrm{PANI} / \mathrm{CC} / \mathrm{MnO}_{2}$ with highly porous and ordered structure significantly increases the efficient contact surface and shortens the pathway for ions and electron transport, which shows an exceptionally high specific capacitance, long term cycle stability, higher energy density and power density. Taken together, our developed PANI/CC hybrid electrode materials have a promising potential in applications for supercapacitors.

\section{Acknowledgement}

This work was supported by State Key Laboratory on Integrated Optoelectronics, College of Electronic Science and Engineering, Jilin 
University and the National Science Foundation of China (No. 61474056 and 61374218).

\section{Appendix A. Supplementary data}

Supplementary data to this article can be found online at http://dx. doi.org/10.1016/j.jelechem.2017.03.029.

\section{References}

[1] R. Kötz, R. Kötz, M. Carlen, M. Carlen, Principles and applications of electrochemical capacitors, Electrochim. Acta 45 (2000) 2483-2498, http://dx.doi.org/10.1016/ S0013-4686(00)00354-6.

[2] B.E. Conway, V. Birss, J. Wojtowicz, The role and utilization of pseudocapacitance for energy storage by supercapacitors, J. Power Sources 66 (1997) 1-14, http://dx.doi. org/10.1016/S0378-7753(96)02474-3.

[3] C. Liu, Z. Yu, D. Neff, A. Zhamu, B.Z. Jang, Graphene-based supercapacitor with an ultrahigh energy density, Nano Lett. 10 (2010) 4863-4868, http://dx.doi.org/10.1021/ nl102661q.

[4] S. He, L. Chen, C. Xie, H. Hu, S. Chen, M. Hanif, et al., Supercapacitors based on 3D network of activated carbon nanowhiskers wrapped-on graphitized electrospun nanofibers, J. Power Sources 243 (2013) 880-886, http://dx.doi.org/10.1016/j. jpowsour.2013.06.104.

[5] C.-H. Wang, H.-C. Hsu, J.-H. Hu, High-energy asymmetric supercapacitor based on petal-shaped $\mathrm{MnO} 2$ nanosheet and carbon nanotube-embedded polyacrylonitrilebased carbon nanofiber working at $2 \mathrm{~V}$ in aqueous neutral electrolyte, J. Power Sources 249 (2014) 1-8, http://dx.doi.org/10.1016/j.jpowsour.2013.10.068.

[6] P. Simon, Y. Gogotsi, Materials for electrochemical capacitors, Nat. Mater. 7 (2008) 845-854, http://dx.doi.org/10.1038/nmat2297.

[7] G.A. Snook, P. Kao, A.S. Best, Conducting-polymer-based supercapacitor devices and electrodes, J. Power Sources 196 (2011) 1-12, http://dx.doi.org/10.1016/j.jpowsour. 2010.06.084.

[8] C. Xia, Y. Xie, Y. Wang, W. Wang, H. Du, F. Tian, Preparation and capacitance performance of polyaniline/titanium nitride nanotube hybrid, J. Appl. Electrochem. 43 (2013) 1225-1233, http://dx.doi.org/10.1007/s10800-013-0610-x.

[9] C. Xia, Y. Xie, W. Wang, H. Du, Fabrication and electrochemical capacitance of polyaniline/titanium nitride core-shell nanowire arrays, Synth. Met. 192 (2014) 93-100, http://dx.doi.org/10.1016/j.synthmet.2014.03.018.

[10] M. Mastragostino, C. Arbizzani, F. Soavi, Polymer-based supercapacitors, J. Power Sources 97-98 (2001) 812-815, http://dx.doi.org/10.1016/S0378-7753(01)00613-9.

[11] M. Wan, Some issues related to polyaniline micro-/nanostructures, Macromol. Rapid Commun. 30 (2009) 963-975, http://dx.doi.org/10.1002/marc.200800817.

[12] J. Stejskal, P. Kratochvı, S.P. Armes, S.F. Lascelles, Polyaniline dispersions. 6. † Stabilization by colloidal silica particles Andrea Riede and Martin Helmstedt ${ }^{`}$ ivka, Macromolecules 9297 (1996) 6814-6819, http://dx.doi.org/10.1021/ma9603903.
[13] S. Ameen, M.S. Akhtar, H.S. Shin, A sea-cucumber-like hollow polyaniline spheres electrode-based chemical sensor for the efficient detection of aliphatic alcohols, RSC Adv. 3 (2013) 10460-10,470, http://dx.doi.org/10.1039/c3ra00016h.

[14] Z. Wei, M. Wan, Hollow microspheres of polyaniline synthesized with an aniline emulsion template, Adv. Mater. 14 (2002) 1314-1317, http://dx.doi.org/10.1002 1521-4095(20020916)14:18 < 1314::AID-ADMA1314 > 3.0.CO;2-9.

[15] J. Shen, C. Yang, X. Li, G. Wang, High-performance asymmetric supercapacitor based on nanoarchitectured polyaniline/graphene/carbon nanotube and activated graphene electrodes, ACS Appl. Mater. Interfaces 5 (2013) 8467-8476, http://dx. doi.org/10.1021/am4028235.

[16] H. Cao, X. Zhou, Y. Zhang, L. Chen, Z. Liu, Microspherical polyaniline/graphene nanocomposites for high performance supercapacitors, J. Power Sources 243 (2013) 715-720, http://dx.doi.org/10.1016/j.jpowsour.2013.06.032.

[17] Q. Li, J. Liu, J. Zou, A. Chunder, Y. Chen, L. Zhai, Synthesis and electrochemical performance of multi-walled carbon nanotube/polyaniline/MnO2 ternary coaxial nanostructures for supercapacitors, J. Power Sources 196 (2011) 565-572, http://dx. doi.org/10.1016/j.jpowsour.2010.06.073.

[18] Z. Niu, W. Zhou, X. Chen, J. Chen, S. Xie, Highly compressible and all-solid-state supercapacitors based on nanostructured composite sponge, Adv. Mater. (2015) n/a-n/a 10.1002/adma.201502263.

[19] K. Oyaizu, Excitation; coupling to other species (such as methane), the coupling will be, Science 80 (319) (2008) 737-738.

[20] V.L. Pushparaj, M.M. Shaijumon, A. Kumar, S. Murugesan, L. Ci, R. Vajtai, et al., Flexible energy storage devices based on nanocomposite paper, Proc. Natl. Acad. Sci. U. S. A. 104 (2007) 13574-13,577, http://dx.doi.org/10.1073/pnas.0706508104.

[21] Y. Lee, C. Chang, S. Yau, L. Fan, Y. Yang, L.O. Yang, et al., Conformations of polyaniline molecules adsorbed on Au(111) probed by in situ STM and ex situ XPS and NEXAFS, J. Am. Chem. Soc. 131 (2009) 6468-6474, http://dx.doi.org/10.1021/ja809263y.

[22] J. Xu, K. Wang, S.Z. Zu, B.H. Han, Z. Wei, Hierarchical nanocomposites of polyaniline nanowire arrays on graphene oxide sheets with synergistic effect for energy storage, ACS Nano 4 (2010) 5019-5026, http://dx.doi.org/10.1021/nn1006539.

[23] W. Focke, G. Wnek, Y. Wei, Influence of oxidation state, $\mathrm{pH}$, and counterion on the conductivity of polyaniline, J. Phys. Chem. 91 (1987) 5813-5818, http://dx.doi.org/ 10.1021/j100306a059.

[24] Y. Li, X. Zhao, Q. Xu, Q. Zhang, D. Chen, Facile preparation and enhanced capacitance of the polyaniline/sodium alginate nanofiber network for supercapacitors, Langmuir 27 (2011) 6458-6463, http://dx.doi.org/10.1021/la2003063.

[25] M. Sasidharan, N.K. Mal, A. Bhaumik, In-situ polymerization of grafted aniline in the channels of mesoporous silica SBA-15, J. Mater. Chem. 17 (2007) 278, http://dx.doi. org $/ 10.1039 / \mathrm{b} 610392 \mathrm{~h}$

[26] V. Sahu, S. Goel, R.K. Sharma, G. Singh, Zinc oxide nanoring embedded lacey graphene nanoribbons in symmetric/asymmetric electrochemical capacitive energy storage, Nanoscale 7 (2015) 20642-20,651, http://dx.doi.org/10.1039/ C5NR06083D. 